\title{
Ataxia and Diplopia in a Patient with Chronic Lymphocytic Leukemia
}

\author{
Teneille E. Gofton, David R. Macdonald, M. Carmela Tartaglia, \\ Donald H. Lee, Joseph F. Megyesi, Robert R. Hammond
}

Can. J. Neurol. Sci. 2008; 35: 243-246

\section{Clinical Presentation - Dr. Gofton}

A female in her early 60 's presented with a four month history of double vision and frequent falls. The falls had increased over the two weeks prior to presentation leading to bruises of varying ages on her right side and an abrasion below her right orbit. The patient's family had also noticed confusion and slowed speech.

The patient's past medical history was significant for a nine year history of chronic lymphocytic leukemia (CLL), a hysterectomy and oophorectomy for benign disease, carpal tunnel syndrome, depression and elevated cholesterol. She was a nonsmoker and did not consume alcohol. There was no significant family history or consanguinity. There was no recent travel.

Medications at the time of presentation consisted of: paroxetine $20 \mathrm{mg}$ po once daily, vitamin B1 po once daily, atarvastatin $20 \mathrm{mg}$ po once daily, acetylsalicylic acid $81 \mathrm{mg}$ po once daily and chlorambucil $2 \mathrm{mg}$ po bid.

At the time of presentation, the patient had normal vital signs and was in no acute distress. Systemic examination was unremarkable and there was no lymphadenopathy. Examination of the cranial nerves revealed right esotropia and hypometric saccades to the right. The visual fields and fundi were normal. The remainder of the cranial nerve examination was unremarkable. Motor examination revealed normal bulk, tone and power. Sensory examination was normal in all modalities. Reflexes were $2+$ and symmetrical. The plantar response was downgoing on the left and upgoing on the right. Examination of gait demonstrated a high stepping gait on the right, falls to the right with ambulation and an inability to perform tandem gait. Right arm dysmetria was present on finger-nose testing and there was dysdiadochokinesia on the right side.

\section{Case Discussion - Dr. Macdonald}

Localization of the case presented above is based on several signs and symptoms. The main complaints at presentation were diplopia and falls to the right. Diplopia localizes to the brainstem and may be caused by a IIIrd, IVth or VIth cranial nerve lesion in the midbrain and pons or along the course of the nerve in the posterior fossa, cavernous sinus or orbit. Falls to the right could localize to the right cerebellum or to the brainstem connections to the right cerebellum. Falls to the right could also be accounted for by a right hemiparesis with anomalies in the left hemisphere or its connections. The slowed speech, which was part of the presenting symptoms, may be caused either by left frontal pathology if there is an element of aphasia or by a cerebellar or brainstem lesion if there is dysarthria. At this time, the confusion is not of localizing value as it remains subjective and there is a normal mental status examination.

The right esotropia and the hypometric saccades suggest involvement of the right brainstem and the paramedian pontine reticular formation. The right sided dysmetria and dysdiadochokinesia localize to the cerebellum or its brainstem connections. An extensor plantar response on the right indicates corticospinal tract involvement either within the right spinal cord, the left brainstem, cerebral peduncle, internal capsule or frontal lobe.

Given these signs and symptoms, the localization is most likely to include a lesion affecting the brain-stem and right cerebellum or cerebellar connections. However, the absence of other cranial nerve findings along with normal power and sensation make a large brainstem lesion less likely. The cause of the signs and symptoms is likely multifocal and there are no signs or symptoms suggestive of raised intracranial pressure.

Due to the progressive nature of the presentation, a vascular or traumatic event is unlikely to be the cause. The history is also too long and there is a paucity of associated symptoms such as fever and headache to favour an infectious etiology. The history is also quite prolonged for a neoplastic process such as brain or leptomeningeal metastases from a previously undiagnosed systemic cancer. On the other hand, the history is too short to be compatible with a degenerative process and there is also a lack of focal findings. There is the potential for these symptoms to be drug-related (on chlorambucil for nine years), but the dose of chlorambucil is relatively low and no other side effects had been noted.

From the Departments of Pathology (RRH), Clinical Neurological Sciences (TEG, DRM, MCT, DHL, JFM, RRH), Oncology (DRM) and Diagnostic Radiology (DHL), London

Health Sciences Centre and the University of Western Ontario, London, Ontario, Canada.

Received August 29, 2007. Final Revisions Submitted December 17, 2007.

Reprint requests to: R.R. Hammond, Department of Pathology, LHSC-UH, 339 Windermere Road, London, Ontario, N6A 5A5, Canada. 
The differential diagnosis is broad at this point and includes: i. a neoplastic process (such as a primary CNS glioma or lymphoma, CNS involvement by CLL, intravascular lymphoma), ii. a chronic opportunistic infectious process (progressive multifocal leukoencephalopathy (PML), chronic meningitis), iii. trauma (chronic subdural hematoma) or iv. an inflammatory process (CNS vasculitis, paraneoplastic process).

\section{Clinical Presentation - Dr. Gofton}

Initial investigations showed a white blood cell count of 45.4 x $10^{9} / \mathrm{L}$ (predominantly lymphocytes with "smudge" cells noted), a hemoglobin of $111 \mathrm{~g} / \mathrm{L}$ and a platelet count of $249 \times 10^{9} / \mathrm{L}$. Serum flow cytometry demonstrated a monoclonal B cell population consistent with CLL. The erythrocyte sedimentation rate was elevated at $47 \mathrm{~mm} / \mathrm{hr}$ and $\mathrm{C}$ reactive protein was within normal limits. Analysis of the complement system showed an elevated $\mathrm{CH} 50$ at 94 (upper limit of normal for laboratory 80) and normal levels of $\mathrm{C} 3$ and $\mathrm{C} 4$. A vasculitic screen revealed normal levels of ANA, anti-dsDNA, anti-ENA, p-ANCA and cANCA. No monoclonal proteins were present on serum electrophoresis. Hepatitis B, hepatitis C and HIV testing were negative. A serum paraneoplastic screening panel was negative.

A lumbar puncture was performed. The cerebrospinal fluid was clear and colourless. Tube 1 had four red blood cells and three nucleated cells (72\% lymphocytes). Tube 2 had a glucose of $3.1 \mathrm{mmol} / \mathrm{L}$ (serum glucose 5.1) and a protein of $278 \mathrm{mg} / \mathrm{L}$.
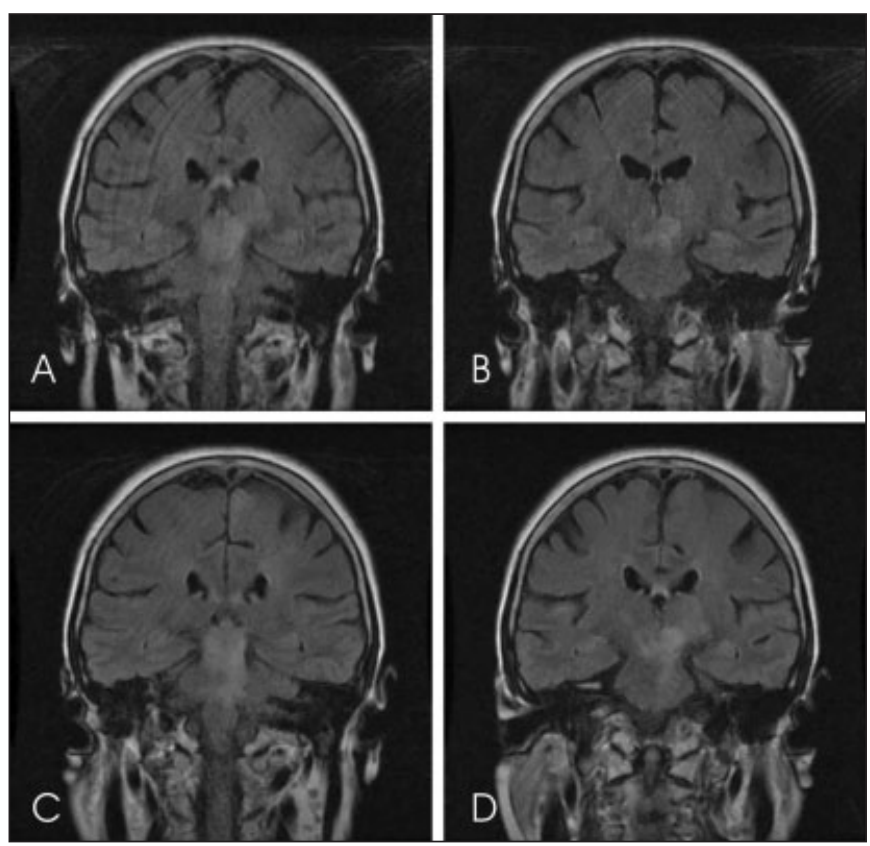

Figure 1: $A$ and B) Coronal MRI FLAIR images at the patient's initial presentation showing irregular areas of increased signal in the diencephalon, midbrain and pons. C and D) Coronal MRI FLAIR images on readmission showing progression of signal changes in diencephalon, midbrain and pons with patchy increased signal also noted in the right insular and left frontal cortices.
Tube 4 had six red blood cells and one nucleated cell. Cerebrospinal fluid flow cytometry was unremarkable. The cerebrospinal fluid was also negative for Epstein-Barr virus, cytomegalovirus, arbovirus, bacteria, acid fast bacilli, Borrelia and cryptococcal antigen.

Mammography and chest imaging were normal and an abdominal/pelvic ultrasound confirmed her previous hysterectomy and oophorectomy with no further abnormalities.

Magnetic resonance imaging of the brain (Figure 1) demonstrated increased signal on T2-weighted images in the right temporal subcortical white matter, left thalamus and cerebral peduncle, midbrain tectum and pontine tegmentum. There was no mass effect and the grey matter was relatively spared. There was no change following gadolinium administration and no diffusion weighted abnormalities were seen.

\section{Case Discussion - Dr. Macdonald}

The clinical investigations and imaging performed virtually exclude a primary neoplastic process (no enhancement or mass effect) and CNS involvement by CLL (no enhancement, normal CSF and no evidence of high grade non-Hodgkin's lymphoma). The normal CSF also excludes the possibility of a chronic meningitic process, no subdural is seen on imaging and there is no evidence of an inflammatory process such as CNS vasculitis. There is no evidence of systemic cancer and the presenting symptoms are not suggestive of a typical paraneoplastic syndrome (similarly, the paraneoplastic screen was negative).

On the other hand, these investigations do not rule out a multifocal low-grade glioma or gliomatosis. Such a diagnosis would be unusual at this age and there is no mass effect on imaging. The possibility of intravascular lymphoma remains but there are no systemic signs or symptoms, the CSF is normal and there are no diffusion-weighted (DWI) or post gadolinium changes seen on MRI. The possibility of a chronic multifocal infection such as PML remains strong.

\section{Clinical Presentation - Dr. Gofton}

The patient was discharged home after one week in hospital but was readmitted three weeks later with a history of worsening diplopia, gait instability and falls. There was also significant dysarthria and dysphagia. Upon examination, new findings included bilateral sixth nerve palsies and impairment of upward gaze. She was dysarthric, had right hemiparesis and right sided dysmetria. Reflexes were brisk throughout, but symmetrical and the plantar responses were unchanged. Sensory examination remained normal in all modalities.

Her white count and other laboratory findings were unchanged. An MRI was repeated demonstrating a progression of the previously described abnormalities (Figure 1). A brain biopsy was performed.

Over the following days, the patient developed pneumonia and succumbed to progressive respiratory failure.

\section{Case Discussion - Dr. Macdonald}

In the interim, the patient has demonstrated deterioration of her clinical status over a relatively short period of time. The localization of the patient's symptoms can be largely accomodated in the brainstem (midbrain and pons) and is multifocal in nature. The most likely differential diagnosis is PML, intravascular lymphoma or gliomatosis cerebri. 


\section{Neuropathological Diagnosis - Dr. Tartaglia}

A biopsy of lesional tissue was obtained from the left thalamus under stereotactic guidance. Hematoxylin and eosin (H\&E) stains revealed a lesion with pallor, vacuolation, light perivascular mononuclear infiltrates, activated microglia and a pleomorphic gliosis (Figures 2A and 2B). Many oligodendroglial nuclei were enlarged with chromatin displaced by ground-glass eosinophilic inclusions. Many inclusions virtually filled nuclei and at times displayed polygonal profiles. Staining for glial fibrillary acidic protein (GFAP) revealed scattered large pleomorphic and multinucleated astrocytes with atypical hyperchromatic nuclei (Figure 2C). Simian Virus 40 immunohistochemistry revealed strong labeling of the enlarged inclusion-bearing oligodendrocyte nuclei. (Figure 2D) Electron microscopy confirmed the oligodendroglial nuclear inclusions to consist of crystal-like arrays of viral capsids compatible with polyoma virus (Figure 2E). The final neuropathological diagnosis was PML.

\section{Summary AND Review OF PML - Dr. Gofton}

Progressive multifocal leukoencephalopathy was first described in 1958 by Astrom and colleagues. ${ }^{1}$ The JC virus (JCV) was subsequently isolated from infected glial cells in $1965^{2}$ and definitively linked to PML as the causal agent in $1971 .^{3}$

Humans are natural host species for two members of the polyomavirus family: JCV and BKV. so named using the initials of the respective index patients. Both viruses establish subclinical persistent infection in the kidney and peripheral blood in $35-85 \%$ of the population. ${ }^{4}$ The JCV targets the kidney, gastrointestinal tract and B-lymphocytes in bone marrow and peripheral blood. ${ }^{5}$

CD4 lymphocytes are essential for suppression of JCV replication and for activation of macrophages and cytotoxic $\mathrm{T}$ cells (CD8 cells). Cytotoxic T cells can destroy virus laden cells through recognition of foreign viral antigens in the context of MHC class I molecules on the surface of infected cells. ${ }^{5}$

With immune suppression, reactivation of JCV occurs and virus may spread to the central nervous system (CNS) where it can cytolytically infect oligodendrocytes and establish a nonproductive infection in astrocytes. ${ }^{5}$ Studies have demonstrated that JCV requires $\mathrm{N}$-linked glycoprotein containing terminal alpha-(2,6)-linked sialic acid to successfully infect human glial cells. The virus is endocytosed and transported to the nucleus where uncoating occurs exposing the genome for early gene expression. ${ }^{5}$ Viral DNA synthesis precedes the production of the late proteins which facilitate the assembly of new virions.

Evaluation of early PML lesions in the brain reveals multiple perivascular areas of white matter destruction suggestive of hematogenous spread. ${ }^{6}$ The nuclei of infected oligodendrocytes are swollen with eosinophilic ground-glass viral inclusions. Giant and multinucleated astrocytes are typically present and may also display intranuclear viral inclusions. ${ }^{6}$

\section{Epidemiology}

Eighty five percent of patients with PML are HIV positive. Progressive multifocal leukoencephalopathy is the third most common cause of neurological symptoms in AIDS following

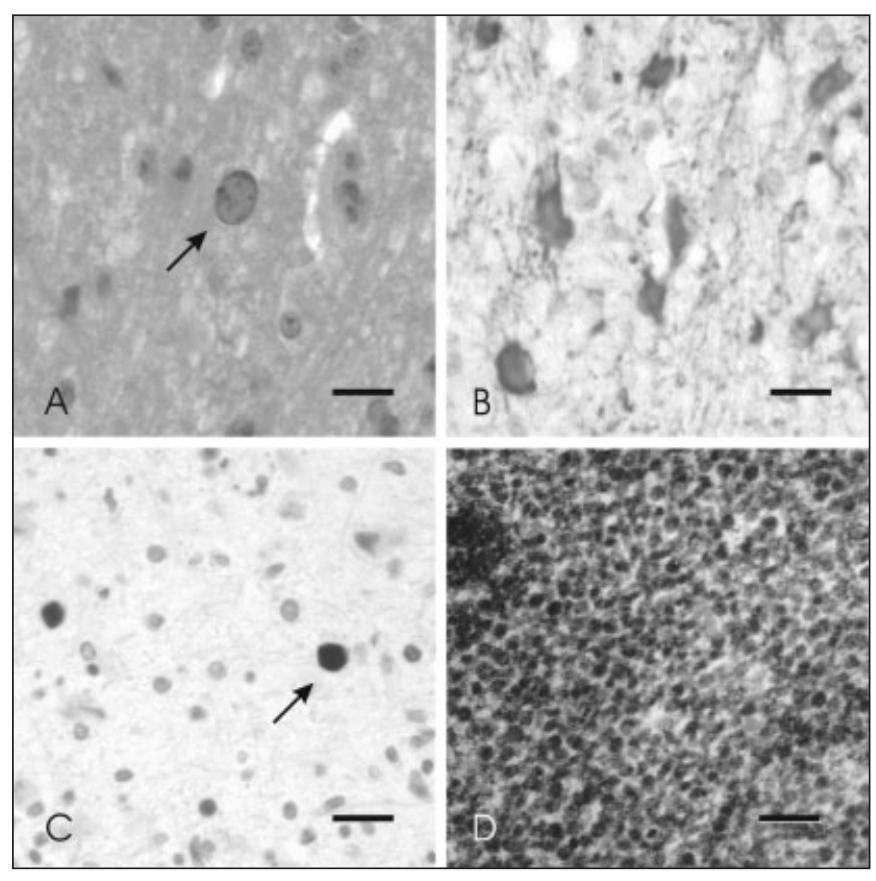

Figure 2: A) Hypertrophied oligodendroglial nucleus with its nucleoplasm largely replaced by an eosinophilic "ground-glass" inclusion. The background neuropil is gliotic and vacuolated. (H\&E, bar =20um). B) Prominent reactive astrocytosis. (anti-GFAP, DAKO: bar=50um). C) Many glial nuclei contained Simian Virus 40 (SV40) antigen, the stained inclusions at times showing a rectilinear profile (arrow). (anti-SV4O, DAKO: bar=50um) D) Ultrastructural examination revealed intranuclear inclusions composed of compact crystalline arrays of $40 \mathrm{~nm}$ diameter round or polygonal particles consistent with JC virus (osmium tetroxide and lead citrate: bar $=100 \mathrm{~nm}$ ). (Note: the sample was retrieved from a formalin-fixed, paraffin-embedded block, post-fixed in glutaraldehyde and processed for electron microscopy)

toxoplasma encephalitis and HIV encephalopathy. ${ }^{7}$ Interestingly, PML is reported to be more common in Europe and North America than in India or Africa, regions that nevertheless have significant populations of HIV infected individuals. ${ }^{8}$ Prior to the HIV pandemic, PML was most commonly reported in the context of lymphoproliferative and myeloproliferative disorders, carcinoma and chronic inflammatory diseases. Case reports in the literature describing PML in the context of hematological malignancies suggest that it typically follows the initiation of cytotoxic therapy. Review of the literature revealed at least two reports of PML arising in the context of CLL. ${ }^{9,10}$

\section{Clinical Presentation}

Reactivation of a latent JCV infection results in the insidious onset of neurologic deficits. The deficits are varied and nonspecific, but most commonly include motor, speech, visual, personality and cognitive disturbances, corresponding to the areas of demyelination. The most common presenting symptoms are weakness, cognitive impairment, speech abnormality, headache, gait impairment, visual abnormality, sensory loss, seizures and limb incoordination. ${ }^{5,7}$ On examination, the most 
commonly encountered clinical signs include hemiparesis, gait disturbance, cognitive impairment, dysarthria, dysphasia, hemisensory loss, visual field defects and ocular palsies..$^{5.7}$

\section{Laboratory Investigations}

Examination of the CSF is usually unremarkable in individuals with PML. Molecular assays can be performed to identify the JCV in the CSF. ${ }^{5.6 .7}$ Neuroimaging is usually suggestive of the diagnosis of PML. White matter lesions appear as areas of hypodensity on computed tomography of the brain. On magnetic resonance imaging, white matter lesions are hypointense on T1-weighted images, hyperintense on T2weighted images or fluid attenuated inversion recovery (FLAIR) and there is no or minimal enhancement. ${ }^{6}$ There is no mass effect except in the setting of immune reconstitution inflammatory syndrome.

\section{Diagnosis}

The diagnosis of PML must be considered in the setting of neurological deficits worsening over time with neuroimaging suggestive of a progressive white matter process. Polymerase chain reaction (PCR) DNA amplification for the detection of JCV in CSF can be helpful. Nonetheless, the gold standard for the diagnosis remains pathological evidence of infection. Neuropathological examination must be carried out with a high index of suspicion as the diagnosis may not have been raised in the preoperative differential. Immunohistochemistry, electron microscopy and PCR (even on formalin-fixed tissue) can confirm histological suspicions. ${ }^{6}$ The present case was also confirmed by PCR from the formalin-fixed biopsy material.

\section{Management}

There is no primary treatment for PML. To date, the most successful interventions have included reconstitution of cellmediated immunity using various approaches depending on the underlying cause of immune dysfunction. In some patients, reversal of cell-mediated immune deficiency leads to stabilization of neurological symptoms and resolution of PML. 5,7,11 Institution of highly active antiretroviral therapy (HAART) in the setting of AIDS can lead to a rejuvenated lymphocytic inflammatory reaction that may transiently exacerbate the clinical picture. ${ }^{11}$ Other therapies attempted with limited patient tolerance include topothecan, a topoisomerase inhibitor, and cidofocir, an antiviral agent effective against cytomegalovirus.

\section{Prognosis}

Because there is no specific treatment for PML, it remains a severe and often fatal opportunistic infection. Mortality is as high as $30-50 \%$, however, some patients survive for many years. ${ }^{7}$ Favourable prognostic factors include and a CD4 count greater than 100 cells/ $\mu$ l and starting HAART in HAART naïve patients at the time of diagnosis. ${ }^{11}$ Damaged oligodendrocytes are not effectively replaced, therefore, survivors are often left with significant disability despite control of the viral infection. ${ }^{6}$

\section{Case Summary}

The present case was typical of PML in many respects. The patient experienced an insidious and progressive neurological decline in the setting of impaired immune function. Imaging was consistent with and pathology diagnostic of PML. The present case serves to remind us of the importance of a high index of suspicion in such presentations. The efficient diagnosis of PML can greatly reduce its morbidity and mortality in those patients amenable to treatment.

\section{REFERENCES}

1. Astrom KE, Mancall EL, Richardson EL. Prograssive multifocal leukoencephalopathy; a hitherto unrecognized complication of chronic lymphatic leukemia and Hodgkin's Disease. Brain. 1958;81(1):93-111.

2. Zu Rhein GM, Chou SM. Particles resembling papovaviruses in human cerebral demyelinating disease. Science. 1965;148:1477-9.

3. Padgett B, Zu Rhein GM, Walker D, Echroade R, Dessel B. Cultivation of papova-like virus from human brain with progressive multifocal leukoencephalopathy. Lancet. 1971; 1(7712):1257-60.

4. Knowles WA, Pipkin P, Andrews N, Vyse A, Minor P, Brown DW, et al. Population-based study of antibody to the human polyomaviruses $\mathrm{BKV}$ and $\mathrm{JCV}$ and the simian polyomavirus SV40. J Med Virol. 2003;71:115-23.

5. Eash S, Manley K, Gasparovic M, Querbes W, Atwood WJ. The human polyomaviruses. Cell Mol Life Sci. 2006;63:865-76.

6. Love S. Demyelinating diseases. J Clin Path. 2006;59:1151-9.

7. Koralnik IJ. New insights into progressive multifocal leukoencephalopathy. Curr Opin Neurol. 2004;17: 365-70.

8. Shankar SK, Satishchandra P, Mahadevan A, Yasha TC, Nagaraja $\mathrm{D}$, Taly $\mathrm{AB}$, et al. Low prevalence of progressive multifocal leukoencephalopathy in India and Africa: is there a biological explanation? J Neurovirol. 2003;9 Suppl 1:59-67.

9. Kiewe P, Seyfert S, Korper S, Rieger K, Thiel E, Knauf W. Progressive multifocal leukoencephalopathy with detection of JC virus in a patient with chronic lymphocytic leukemia parallel to onset of fludarabine therapy. Leuk Lymphoma. 2003; 44(10):1815-8

10. Shafran B, Roke ME, Barr RM, Cairncross JG. Contrast enhancing lesions in progressive multifocal leukoencephalopathy: a clinical pathologic correlation. Can J Neurol Sci. 1987;14(4):600-2.

11. Berger JR, Houff S. Progressive multifocal leukoencephalopathy: lessons from AIDS and natalizumab. Neurol Res. 2006;28: 299-305. 